\title{
Heidelberg Edge Perimetry for the Detection of Early Glaucomatous Damage: A Case Report
}

\author{
Amir H. Marvasti, ${ }^{a}$ Andrew J. Tatham ${ }^{a} \quad$ Robert N. Weinreb ${ }^{a}$ \\ Felipe A. Medeiros ${ }^{a}$ \\ ${ }^{a}$ Hamilton Glaucoma Center, Department of Ophthalmology, University of California San \\ Diego, La Jolla, Calif., and ${ }^{\mathrm{b}}$ Boston University School of Medicine, Boston, Mass., USA
}

\section{Key Words}

Heidelberg edge perimetry · Glaucoma $\cdot$ Raised intraocular pressure $\cdot$ Standard automated perimetry · Ocular hypertension

\begin{abstract}
Purpose: To illustrate the potential role for Heidelberg edge perimetry (HEP) in the assessment of early functional loss in glaucoma. Case Report: A 46-year-old male presented with raised intraocular pressure but healthy optic discs and normal standard automated perimetry (SAP). He was diagnosed with ocular hypertension, but after 16 years, he developed glaucomatous changes to the right optic disc and inferior retinal nerve fiber layer thinning. Despite the evidence of progressive structural damage, functional testing using SAP was repeatedly normal, based on conventional criteria. HEP, on the other hand, revealed an extensive superior visual field damage in agreement with the structural changes. Additionally, and in contrast to SAP, the HEP indices of Glaucoma Hemifield Test, mean deviation and pattern standard deviation were all outside normal limits. Conclusion: This case illustrates that HEP may have an important role in the early detection of functional damage in glaucoma, with potential advantages over SAP.

(c) 2013 S. Karger AG, Basel
\end{abstract}

\section{Introduction}

Glaucoma is a heterogeneous group of potentially blinding disorders characterized by progressive optic neuropathy. Given the irreversible nature of this disease, early diagnosis and detection of progression is important, particularly as appropriate treatment can slow or halt visual loss.

Felipe A. Medeiros, MD, PhD

Hamilton Glaucoma Center

University of California San Diego

9500 Gilman Drive, La Jolla, CA 92093-0946 (USA)

E-Mail fmedeiros@glaucoma.ucsd.edu 
Marvasti et al:: Heidelberg Edge Perimetry for the Detection of Early Glaucomatous Damage: A Case Report

The assessment of visual function is an essential component of glaucoma diagnosis and monitoring, with the current gold standard for visual function testing being standard automated perimetry (SAP). However, qualitative and quantitative analyses of the optic nerve and retinal nerve fiber layer (RNFL) have shown that significant structural changes are present in many patients before detectable changes in SAP [1]. As such, sole reliance on SAP, especially in early stages of the disease, has the potential for underestimating both neural loss and risk of functional impairment [2].

Based on these observations, new perimetric techniques have been developed with the goal of detecting glaucomatous functional damage at an earlier stage. One recently developed device is the Heidelberg Edge Perimeter [3], which uses a flicker-defined form (FDF) stimulus. FDF perimetry employs a temporally driven illusion within which an array of randomly positioned black and white dots flicker at high temporal frequency in counterphase. The spots reverse polarity without changing their position, which means that white dots are replaced with black dots and black dots are replaced with white dots. At high temporal frequency, subjects perceive an illusory circular edge contour, which appears as a gray patch against the mean luminance background. This stimulus is thought to stimulate the magnocellular pathway, which is considered to be damaged in early stages of glaucoma [4]. Although the exact mechanism underlying the FDF stimulus and its effect on the magnocellular pathway have not yet been fully elucidated, there is some evidence to suggest that FDF perimetry could be more sensitive at detecting early functional loss than SAP and other types of perimetry $[5,6]$.

The following report illustrates the potential role of Heidelberg edge perimetry (HEP) in the assessment of early functional loss in a case of confirmed glaucomatous structural damage in the absence of a significant SAP defect.

\section{Case Report}

This is a case of an asymptomatic male, who originally presented in 1993, at age 46, due to an incidental finding of raised intraocular pressure (IOP). There was no family history of glaucoma and no significant past medical or ocular problem aside from low hyperopia (OD: $+1.00 /+0.25 \times 155$, OS: $+1.25 /+0.50 \times 30$ ). There was also no history of systemic medication or corticosteroid use. On Goldmann applanation tonometry, IOPs were $27 \mathrm{~mm} \mathrm{Hg}$ in the right eye (OD) and $24 \mathrm{~mm} \mathrm{Hg}$ in the left eye (OS). Central corneal thickness was $570 \mu \mathrm{m}$ OD and $567 \mu \mathrm{m}$ OS. Gonioscopy revealed open angles in both eyes. On dilated fundoscopic examination, both optic discs appeared healthy and there were no RNFL defects visible. SAP was normal in both eyes. The patient was diagnosed with ocular hypertension and the decision was made to monitor him without treatment.

For the next 16 years, IOPs were consistently in the mid-teens and optic disc and SAP parameters remained normal; however, in 2009, changes to the right optic disc were noted (fig. 1). There was an increase in the excavation of the right disc with a thinning of the inferior neuroretinal rim, and an optic disc hemorrhage was present inferiorly. IOPs had increased to $40 \mathrm{~mm} \mathrm{Hg}$ OD and $30 \mathrm{~mm} \mathrm{Hg}$ OS. A structural abnormality of the right eye was confirmed with spectral domain optical coherence tomography (SD-OCT).

The patient was commenced on topical ocular antihypertensive treatment in both eyes; however, reduction in IOP was insufficient. He had a stepwise increase in treatment and, by 2012, was using 3 antiglaucoma medications (Brinzolamide t.i.d., Timolol once/daily and Latanoprost q.h.s. to both eyes). He also underwent 360 degree selective laser trabeculoplasty in the right eye. At his most recent visit, IOPs were $19 \mathrm{~mm} \mathrm{Hg} \mathrm{OD}$ and $18 \mathrm{~mm} \mathrm{Hg} \mathrm{OS.}$ 
Marvasti et al:: Heidelberg Edge Perimetry for the Detection of Early Glaucomatous Damage: A Case Report

Despite the glaucomatous optic disc changes in the right eye, SAP global indices were within statistically normal limits (fig. 3b).

Due to the apparent mismatch between structural and functional damage, the patient underwent HEP to determine whether functional damage might in fact be present. HEP revealed an extensive abnormality, with a marked nasal and superior paracentral scotoma (fig. 2). In contrast to the SAP indices, the HEP indices of the Glaucoma Hemifield Test (GHT), mean deviation (MD) and pattern standard deviation (PSD) were all outside normal limits. The HEP MD was $-9.55 \mathrm{~dB}(\mathrm{p}<0.05)$ and the HEP PSD was $5.25 \mathrm{~dB}(\mathrm{p}<0.05)$. Figure 3 compares SAP, frequency doubling technology (FDT) and HEP, which were completed on the same day. Both FDT and HEP detected a statistically significant defect while SAP did not; however, the HEP defect was more extensive than the defect observed on FDT. There was also a good spatial correlation between the defect on the Heidelberg Edge Perimeter and the defect observed on optic disc photographs and SD-OCT (fig. 3).

\section{Discussion}

This was a case of a patient with ocular hypertension who developed glaucoma as documented by structural changes on stereoscopic optic disc photographs and SD-OCT imaging. Despite evidence of progressive structural damage (fig. 1), the SAP global indices GHT, MD and PSD remained within statistically normal limits (fig. 1, fig. 3). In contrast, a marked visual field defect was detected on the Heidelberg Edge Perimeter and the HEP indices GHT, MD and PSD were all abnormal (fig. 2, fig. 3). The difference between SAP and HEP was of particular significance as HEP indicated that the visual field defect involved an area close to fixation.

SAP uses a differential contrast stimulus consisting of a white light presented on a white background. Most subtypes of retinal ganglion cells (RGC) can respond to a white-on-white stimulus and, therefore, due to redundancy in the visual system, loss of a large number of RGC can occur before a SAP defect is detected [7]. Histological studies in humans [8] and monkeys [9] have investigated the relationship between SAP and RGC counts. These studies also indicated that a large reduction in RGC density could occur before significant SAP defects develop. The result is that in early glaucoma, reliance on SAP may lead to an underestimation of the amount of glaucomatous damage.

Functional tests targeting specific subtypes of RGC, with lower levels of redundancy, may be able to detect damage at an earlier stage. Such tests would be particularly effective if they were to target groups of RGC susceptible to early glaucomatous damage [5, 10-12]. Although it is unlikely that any stimulus can be specific for a single visual pathway, it is possible to design stimuli to minimize the potential input from other pathways. HEP uses an FDF stimulus to create a high temporal frequency, contrast detection task. This is a stimulus that likely engages the magnocellular pathway. Previous histopathologic studies have reported that the magnocellular pathway is damaged early in the glaucomatous process, and this might explain why HEP was able to detect a functional abnormality before SAP in our patient $[4,5,12]$.

Other perimetric methods have also been introduced with the goal of earlier detection of glaucomatous visual field loss $[13,14]$; however, a recent study comparing HEP with these techniques reported HEP to be a more sensitive test with a stronger correlation to structural measurements [6]. 
Marvasti et al:: Heidelberg Edge Perimetry for the Detection of Early Glaucomatous Damage: A Case Report

\section{Conclusion}

The aim of this report was to explore the potential use of FDF perimetry in the management of a glaucoma patient with statistically normal SAP results. As demonstrated, HEP detected a clinically and statistically significant perimetric defect and documented the area of damage that other perimetric technologies were not able to detect. There was also a good structure-function correlation with the superior nasal defect detected by HEP, corresponding to the area of the inferior thinning of the neuroretinal rim and RNFL. HEP also indicated a small inferior visual field defect, which was consistent with the thinning of the superior RNFL; the SD-OCT indicated a superior quadrant RNFL thickness of $107 \mu \mathrm{m}$, which although was still within statistically normal limits, was significantly lower than the $132 \mu \mathrm{m}$ thickness in the same sector in the left eye.

This case illustrates that HEP may have an important role in the early detection of functional damage in glaucoma, with potential advantages over SAP. HEP may have the potential to become an important component of our armamentarium for the diagnosis and monitoring of glaucoma; however, prospective longitudinal studies are required to fully validate this technology.

\section{Disclosure Statement}

The author(s) have made the following disclosure(s):

A.H.M. - none.

A.J.T. - none.

R.N.W. - Aerie: financial support; Alcon: consultant; Allergan: consultant; Altheos: consultant; Amakem: consultant; Bausch \& Lomb: consultant; Carl Zeiss-Meditec: consultant; Genentech: financial support; Haag-Streit: financial support; Heidelberg Engineering: financial support; Konan: financial support; Lumenis: financial support; National Eye Institute: financial support; Nidek: financial support; Optovue: consultant; Quark: consultant; Solx: consultant; Topcon: consultant.

F.A.M. - Carl-Zeiss: financial support; Heidelberg Engineering: financial support; Topcon: financial support; Alcon: financial support; Allergan: financial support; Sensimed: financial support; Reichert: financial support.

\section{References}

1 Medeiros FA, Alencar LM, Zangwill LM, Bowd C, Sample PA, Weinreb RN: Prediction of functional loss in glaucoma from progressive optic disc damage. Arch Ophthalmol 2009;127:1250-1256.

$>2$ Medeiros FA, Zangwill LM, Bowd C, Mansouri K, Weinreb RN: The structure and function relationship in glaucoma: implications for detection of progression and measurement of rates of change. Invest Ophthalmol Vis Sci 2012;53:6939-6946.

-3 Quaid PT, Flanagan JG: Defining the limits of flicker defined form: effect of stimulus size, eccentricity and number of random dots. Vision Res 2005;45:1075-1084.

4 Rogers-Ramachandran DC, Ramachandran VS: Psychophysical evidence for boundary and surface systems in human vision. Vision Res 1998;38:71-77.

5 Flanagan JG, Williams-Lyn D, Trope GE, Hatch W, Harrison E: The phantom contour illusion letter test: a new psychophysical test for glaucoma? Perimetry Update 1994/95, pp 405-409.

-6 Lamparter J, Russell RA, Schulze A, Schuff AC, Pfeiffer N, Hoffmann EM: Structure-function relationship between FDF, FDT, SAP, and scanning laser ophthalmoscopy in glaucoma patients. Invest Ophthalmol Vis Sci 2012;53:7553-7559.

7 Shaarawy T, Sherwood MB, Hitchings RA, Crowston JG: Glaucoma. Philadelphia, Saunders Limited, 2009. 
Marvasti et al: Heidelberg Edge Perimetry for the Detection of Early Glaucomatous Damage: A Case Report

-8 Kerrigan-Baumrind LA, Quigley HA, Pease ME, Kerrigan DF, Mitchell RS: Number of ganglion cells in glaucoma eyes compared with threshold visual field tests in the same persons. Invest Ophthalmol Vis Sci 2000;41:741-748.

-9 Harwerth RS, Carter-Dawson L, Shen F, Smith EL, Crawford ML: Ganglion cell losses underlying visual field defects from experimental glaucoma. Invest Ophthalmol Vis Sci 1999;40:2242-2250.

10 Quigley HA, Dunkelberger GR, Green WR: Chronic human glaucoma causing selectively greater loss of large optic nerve fibers. Ophthalmology 1988;95:357-363.

11 Schiller PH, Logothetis NK, Charles ER: Functions of the colour-opponent and broad-band channels of the visual system. Nature 1990;343:68-70.

12 Glovinsky Y: Retinal ganglion cell loss is size dependent in experimental glaucoma. Invest Ophthalmol Vis Sci 1991;32:484-491.

13 Johnson CA, Adams AJ, Casson EJ, Brandt JD: Blue-on-yellow perimetry can predict the development of glaucomatous visual field loss. Arch Ophthalmol 1993;111:645-650.

14 Johnson CA, Samuels SJ: Screening for glaucomatous visual field loss with frequency-doubling perimetry. Invest Ophthalmol Vis Sci 1997;38:413-425.

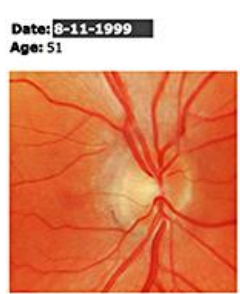

GHT: Within Normal Limits

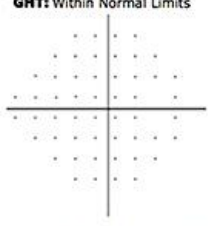

$-1.12 \mathrm{~dB}$ Not Significant
$1.10 \mathrm{~dB}$ Not Significant

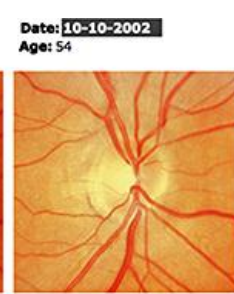

GHT: Within Normal Limits

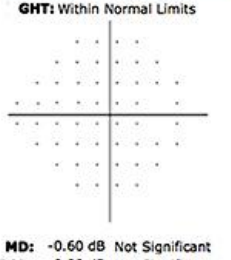

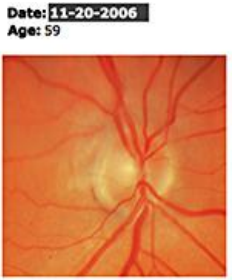

GHT: Within Normal Limits

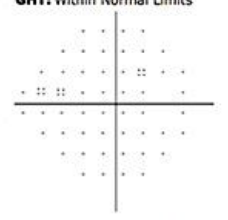

MD: 0.17 AB Not Significant

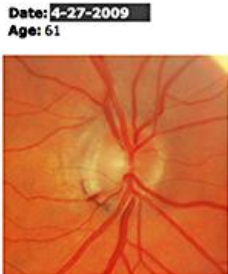

GHT: Within Normal Umits

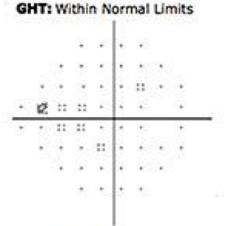

MD: $-0.29 \mathrm{~dB}$ Not Significant

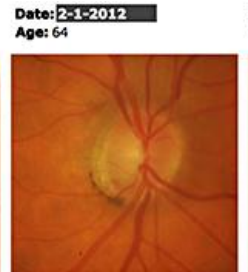

GHT: Within Normal Umits

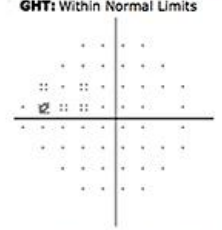

$\begin{array}{ll}\text { MD: } & 0.00 \text { dB Not Significant } \\ \text { PSD: } & 1.60 \text { \&B Not Significant }\end{array}$

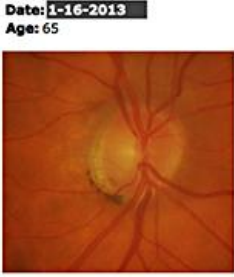

GHT: Within Normal Limits

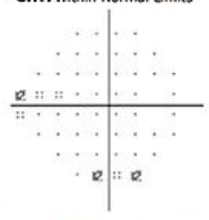

MD: -1.41 dB Not Significan

Fig. 1. Serial optic disc photo and SAP results of the right eye. There is progressive inferior neuroretinal rim thinning over time with an associated optic disc hemorrhage. SAP global indices remained within normal limits throughout the follow-up period. 
Case Reports in

Ophthalmology

a SD-OCT

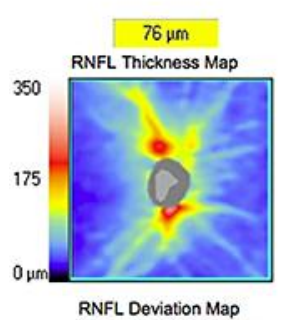

RNFL Deviation Map
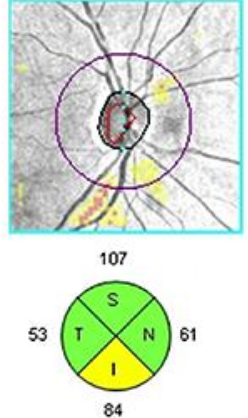

Case Rep Ophthalmol 2013;4:144-150

DOI: $10.1159 / 000355102$
Marvasti et al.: Heidelberg Edge Perimetry for the Detection of Early Glaucomatous Damage: A Case Report

(C) 2013 S. Karger AG, Basel www.karger.com/cop

b SAP
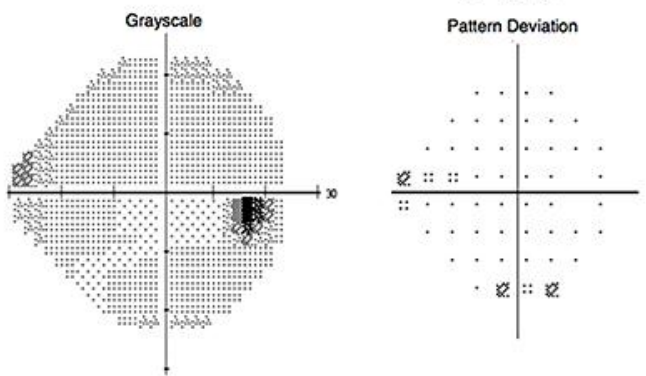

GHT: Within Normal Limits

MD: $-1.41 \mathrm{~dB} \quad$ Not Significant

PSD: $1.89 \mathrm{~dB}<10 \%$

\section{c FDT}

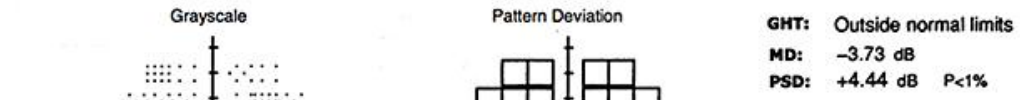

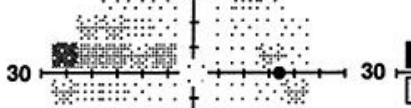
$T^{38} \because$ : : : : : : $:$

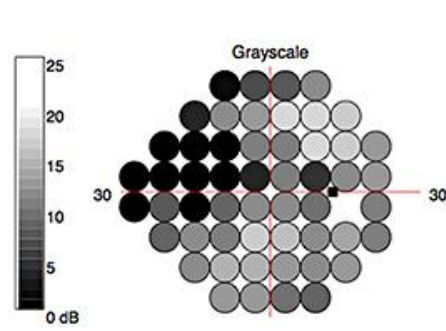

d HEP

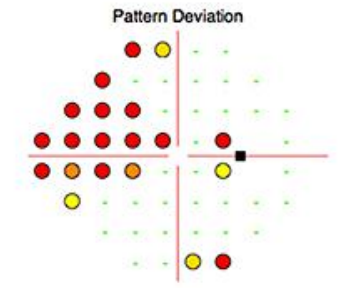

Outside Normal Limits

MD: $\quad-9.55 \mathrm{~dB} \quad p<0.05 \%$

PSD: $+5.25 \mathrm{~dB} \quad p<0.05 \%$

Op<5

Opes

$0 p<1$

Fig. 2. HEP results of the right eye showing extensive superior visual field damage in agreement with optic disc and retinal nerve fiber layer assessment. 


\section{Case Reports in \\ Ophthalmology}

\begin{tabular}{l|l}
\hline Case Rep Ophthalmol 2013;4:144-150 \\
\hline DOI: 10.1159/000355102 & $\begin{array}{l}\text { ○ 2013 S. Karger AG, Basel } \\
\text { www.karger.com/cop }\end{array}$ \\
\hline
\end{tabular}

Marvasti et al.: Heidelberg Edge Perimetry for the Detection of Early Glaucomatous Damage: A Case Report
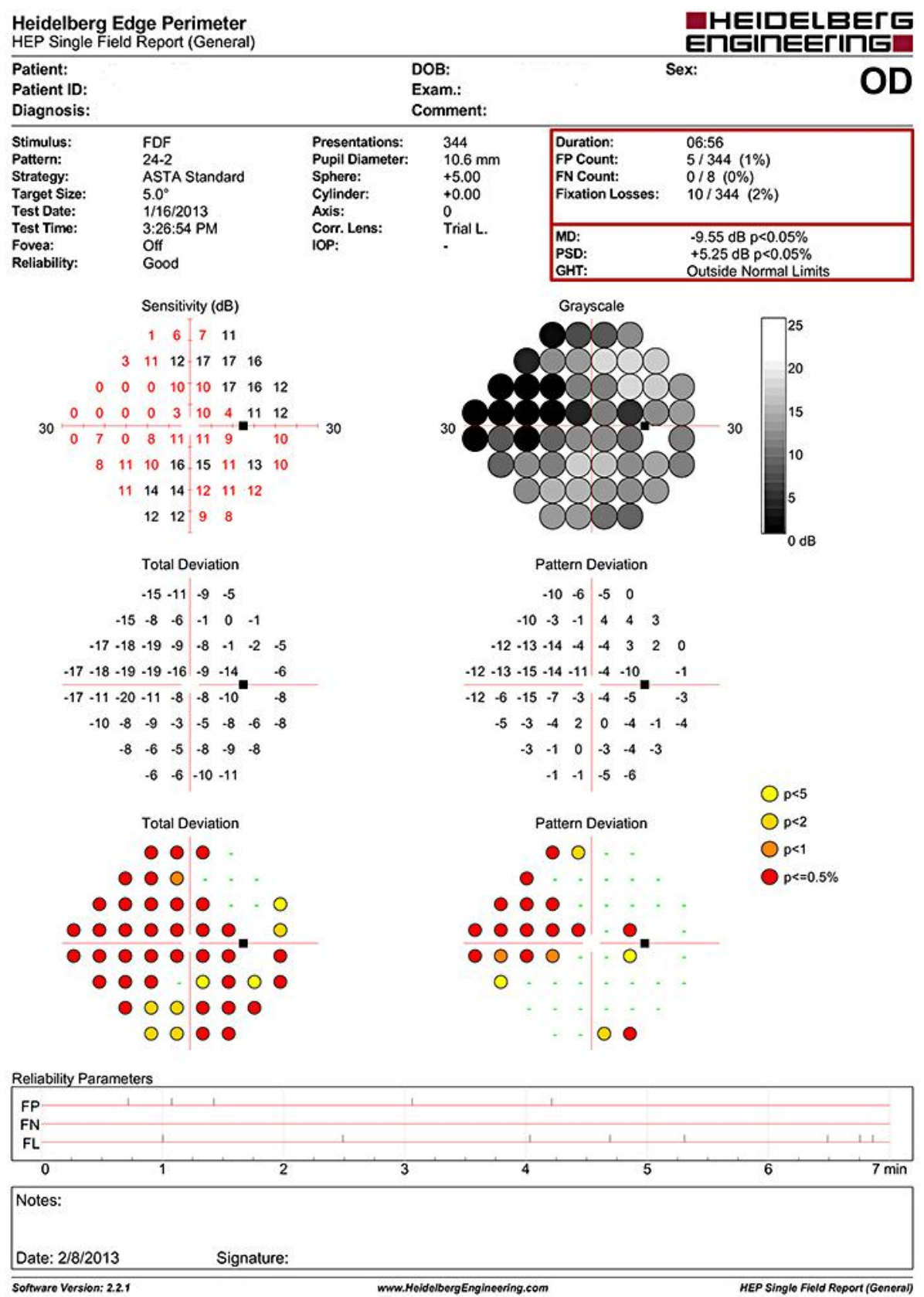

Fig. 3. Comparison between SAP, FDT and HEP. The HEP defect was more extensive than the defect observed on FDT and agreed closely with the retinal nerve fiber layer assessment by SD-OCT. 